\title{
Microbial patterns of Acanthamoeba keratitis at a Singapore ophthalmic referral hospital: A 5-year retrospective observational study
}

\begin{abstract}
Dear Editor,
Acanthamoeba keratitis (AK) is a rare but clinically significant cause of infectious keratitis, its incidence ranging from 0.33 to 1.49 per 10,000 contact lens (CL) wearers. ${ }^{1}$ There is an incomplete understanding of the variable pathogenicity of different strains, leading to diagnostic delays and a lengthy treatment. In this study, we aim to provide an update to the local demographics, clinical and microbiological characteristics of AK.
\end{abstract}

We performed a retrospective review of all corneal scrapings sent for Acanthamoeba species culture at the National University Hospital, a tertiary referral hospital in Singapore, from 1 April 2012 to 31 October 2016. Ethics approval was obtained from the National Healthcare Group Domain Specific Review Board.

A clinical diagnosis of AK was made after verification by a trained corneal specialist and was based on the patient's clinical history and examination findings consistent with AK. ${ }^{2}$ Corneal scraping was performed under a slit-lamp and placed in a transplant medium to be sent to the lab. AK was diagnosed based on a positive culture for Acanthamoeba spp. using nonnutrient agar plate coated with non-mucous bacteria through the entire study period.

Relevant demographic, clinical and treatment information including best-corrected visual acuity, intraocular pressure (IOP), ocular examination findings, and medications were collected by a single person in the team (DC).

Statistical analysis was performed using R statistical software version 3.6.3 (R Project for Statistical Computing). Fisher exact test was performed for categorical variables. Independent t-test or Mann-Whitney $\mathrm{U}$ test were performed for normally and non-normally distributed continuous variables respectively. A $P$ value of less than 0.05 was considered as statistically significant.

A total of 25 corneal scrapings were sent for Acanthamoeba culture. Of these, 6 were repeat scrapings of the same patients, and one did not have available case notes for review. A total of 18 unique patients were included. The mean age was 24.4 years old and two-thirds were female; 14 (77.8\%) were CL wearers and $4(26.7 \%)$ had a history of topical steroid use prior. The median LogMAR visual acuity (VA) was 0.95 and IOP was $16.0 \mathrm{mmHg}$.
Table 1 describes the basic clinical features of patients included in this study. While foreign workers comprised a portion of patients with suspected AK, only one of them eventually had culture-positive AK. Between 2012 and 2015, there were 10 culture-negative suspected AK and one culture-positive AK; in 2016, there were 4 cases of culture-positive AK. There were no statistically significant demographic or clinical differences between culture-positive and culturenegative AKs.

After clinical diagnosis, all patients were started on standard treatment for $\mathrm{AK}$, which included a combination of hexamidine with chlorhexidine eye drops. Ocular steroids were started at a later stage based on clinical response to treatment. Acanthamoeba culture-positive patients all presented with relatively good VA better than 6/24. Two of the culture-positive patients improved after a month of treatment, while 2 worsened. When comparing the presenting VA with the VA at the most recent follow-up, 2 Acanthamoeba culture-positive patients had vision worse than $6 / 120$ at last visit (Patients 19 and 24), despite having good presenting VA (6/9 and 6/15, respectively).

Our study investigated the prevalence of AK over a 5 -year period at the National University Hospital. We found an increase in the number of culture-positive patients in 2016, with all being young female CL wearers.

$\mathrm{AK}$ is an uncommon but sight-threatening form of infectious keratitis. Improper OrthoK lens wear increases the risks. ${ }^{3}$ A previous local study postulated the use of Complete multipurpose solution (Advanced Medical Optics Inc, Santa Ana, US) as a possible cause of an outbreak of AK in 2007. ${ }^{4}$ Diagnosing AK is challenging as early disease has minimal clinical features. In our study, no culture-positive patient had the classical finding of a ring infiltrate, or severe pain out of proportion to clinical signs-a hallmark feature of AK. ${ }^{5}$ Diagnostic modalities include confocal in vivo microscopy, corneal histology, and corneal scraping culture (gold standard), but the yield is often poor due to reduced amoebic density or altered morphology from antimicrobial use. ${ }^{6}$ Polymerase chain reaction is a potential method for diagnosis of patients with reduced amoebic density from antibiotic use, but they are not readily available. Conversely, sometimes the diagnosis of AK cannot be 


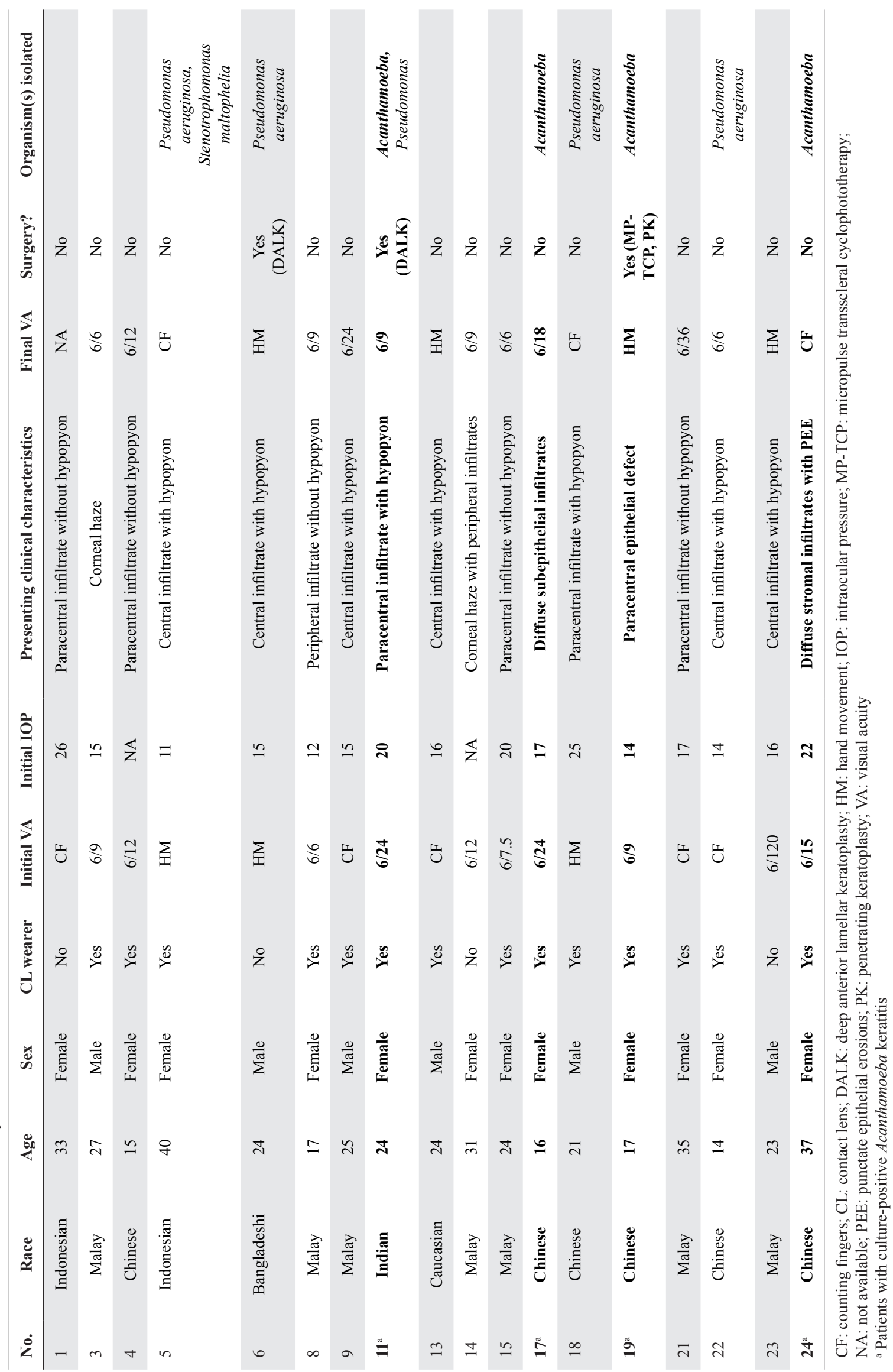


made despite all the clinical features-Patient 15 had all the clinical features of "classic" AK, but her multiple corneal scrapings, confocal microscopy and corneal histology were negative.

Treatment for AK is equally challenging. Standard treatment includes aggressive combined biguanide with aromatic diamidine topical therapy. Most available treatments target the active trophozoites, but are not as effective in eradicating the cystic form. ${ }^{7}$ In our study, despite aggressive combined therapy, Patients 19 and 24 still developed corneal decompensation needing corneal transplants, possibly due to resistance of the cystic form to the antimicrobial agents. For surgical treatment, penetrating keratoplasty is considered if there is persistent inflammation or infection despite maximal antimicrobial therapy. ${ }^{8}$ However, the 1-year survival of penetrating keratoplasty may be as low as $55 \% .{ }^{9}$ In our centre, we prefer deep anterior lamellar keratoplasty (DALK) due to a faster recovery period, lower rejection rate and improved visual outcomes. ${ }^{10}$ Nonetheless, the benefits have to be weighed against the increased risks of residual parasitic load on the corneal endothelial surface after surgery. ${ }^{4}$ For instance, Patient 19 developed endothelial decompensation, which precluded DALK as a viable treatment option.

There are several limitations to our study. Being a retrospective study, data fields were non-standardised. A small sample size, compounded by missing data such as the brands of CL and solution usage precluded further clinical analysis. We also did not perform confocal microscopy in all our patients as this investigation is not routinely available in our centre, which could have resulted in cases of false negatives. Future prospective studies using a standardised data collection form and a standard protocol of workup for AK would help to circumvent this problem.

In conclusion, $\mathrm{AK}$ is an uncommon clinical entity but tends to have significant visual morbidity. Its risk is increased in CL use. The treatment of AK is prolonged and visual outcome is guarded.

\section{REFERENCES}

1. Seal DV, Beattie TK, Tomlinson A, et al. Acanthamoeba keratitis. Br J Ophthalmol 2003;87:516-7.

2. Sun X, Zhang Y, Li R, et al. Acanthamoeba keratitis: Clinical characteristics and management. Ophthalmology 2006;113:412-6.

3. Xuguang S, Lin C, Yan Z, et al. Acanthamoeba keratitis as a complication of orthokeratology. Am J Ophthalmol 2003;136:1159-61.

4. Por YM, Mehta JS, Chua JLL, et al. Acanthamoeba keratitis associated with contact lens wear in Singapore. Am J Ophthalmol 2009; 148:7-12.e2

5. Chynn EW, Lopez MA, Pavan-Langston D, et al. Acanthamoeba keratitis. Contact lens and noncontact lens characteristics. Ophthalmology 1995;102:1369-73.

6. Lam DSC, Houang E, Fan DSP, et al. Incidence and risk factors for microbial keratitis in Hong Kong: Comparison with Europe and North America. Eye (Lond) 2002;16:608-18.

7. Lorenzo-Morales J, Khan NA, Walochnik J. An update on Acanthamoeba keratitis: Diagnosis, pathogenesis and treatment. Parasite 2015;22:10.

8. Cohen EJ, Parlato CJ, Arentsen JJ, et al. Medical and surgical treatment of Acanthamoeba keratitis. Am J Ophthalmol 1987; 103:615-25.

9. Kashiwabuchi RT, Freitas DD, Alvarenga LS, et al. Corneal graft survival after therapeutic keratoplasty for Acanthamoeba keratitis. Acta Ophthalmol 2008;86:666-9.

10. Cheng YYY, Visser N, Schouten JS, et al. Endothelial cell loss and visual outcome of deep anterior lamellar keratoplasty versus penetrating keratoplasty: A randomized multicenter clinical trial. Ophthalmology 2011;118:302-9.

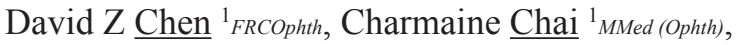
Anna Tan ${ }^{1}$ FAMS, Manotosh Ray ${ }^{1}$ FRCSEd

${ }^{1}$ Department of Ophthalmology, National University Hospital, Singapore

Correspondence: Dr David Chen, Department of Ophthalmology, National University Hospital, 1E Kent Ridge Road, NUHS Tower Block, Level 7, Singapore 119228.

Email: david_chen@nuhs.edu.sg 